\title{
Fatores Prognósticos para o Parto Transvaginal em Pacientes com Cesárea Anterior
}

\author{
Prognostic Factors for Vaginal Delivery \\ after Cesarian Section
}

Luiz Carlos Santos, Melania M. R. Amorim Ana Maria Feitosa Porto, Elvira Barbalho Azevedo, Carina Carla Mesquita

\begin{abstract}
RESUM0
O objetivo deste estudo foi determinar os fatores prognósticos para a ultimação do parto por via transpélvica em gestantes com cesárea anterior admitidas em trabalho de parto no CAMIMIP. Foi realizado um estudo tipo caso-controle, analisando os partos de pacientes com cesárea prévia assistidos no CAM-IMIP no perído de janeiro/ 1991 a dezembro/1994. Foram considerados casos as pacientes com cesárea anterior $(n=156)$ e controles as que tiveram parto transvaginal ( $n=338$ ). Os critérios de inclusão foram: idade gestacional $\geq 36$ semanas, cesárea anterior há pelo menos 1 ano, concepto vivo, trabalho de parto espontâneo $e$ apresentação cefálica fletida. Foram excluidas as gestações de alto risco, os casos de sofrimento fetal anteparto e pacientes com história de parto transpélvico anterior depois da cesárea. A análise estatística foi realizada em Epi-Info 6.0 e Epi-Soft, utilizando-se os testes $\chi^{2}$ de associação, teste exato de Fisher e "t" de Student, bem como a odds ratio e seu intervalo de confiança a 95\%. Análise de regressão logística múltipla foi efetuada para controle das variáveis confundidoras. O percentual de cesáreas no grupo estudado foi de 31,6\%. Os fatores maternos que apresentaram associação significativa com o parto transvaginal foram a idade materna $<20$ anos (OR = 2,07, IC a 95\% = 1,18-3,66) ou $\geq 35$ anos (OR = 0,54, IC a $95 \%=0,36-0,82)$, a história de parto vaginal anterior $(O R=1,6$, IC a $95 \%=1,01-2,55)$ e a indicação da cesárea anterior por doenças da gestação (OR =3,67, IC a 95\% = 1,19-12,02). Fatores como intervalo entre a cesárea anterior e o parto atual, outras indicações de cesárea e o tipo de histerorrafia não apresentaram associação significativa com o parto transpélvico. No modelo de regressão logística múltipla, persistiram como variáveis associadas significativamente ao parto vaginal a idade materna e a história de parto vaginal anterior. Os autores concluíram que sendo a idade materna < 20 anos, a indicação de cesárea por doença da gestação e parto vaginal anterior associaram-se favoravelmente ao parto
\end{abstract}

Instituto Materno-Infantil de Pernambuco (IMIP) - Centro de Atenção à Mulher (CAM)

Correspondência:

Luiz Carlos Santos

IMIP - Rua dos Coelhos, 300 - Ilha do Leite

$50070-530$ - Recife-PE

e-mail: andfpc@br.homeshopping.com.br 
transpélvico em pacientes com cesárea anterior. O risco de cesariana está aumentado em gestantes a partir de 35 anos. Esses fatores devem ser considerados na avaliação obstétrica da via de parto nessas gestantes.

PALAVRAS-CHAVE: Cesárea. Parto normal. Tocurgia. Resolução da gestação.

\section{Introdução}

A alarmante escalada dos índices de cesariana no Brasil constitui um verdadeiro problema de saúde pública. Estima-se que, no país, mais de $40 \%$ dos partos sejam atualmente realizados por via alta, podendo exceder $80 \%$ em algumas regiões e, sobretudo, nos hospitais particulares. Embora a segurança da operação cesariana em nossos dias venha se tornando cada vez maior, vale consignar que a morbimortalidade na cesárea ultrapassa em muito a do parto transpélvico, com o aumento do risco de morte materna variando de 2 a 6 vezes $^{10,13,14}$, além de sérias complicações como a infecção puerperal (risco 8 a 20 vezes maior ${ }^{10,13}$ ), a hemorragia, as embolias $^{10,24}$ e os acidentes anestésicos ${ }^{10}$. Assim, reduzir essas taxas sobrelevadas de cesariana deve representar importante preocupação e, mais que isso, um verdadeiro objetivo da assistência obstétrica.

Nos EUA, na década de 1970 a 1980 houve um aumento na incidência de cesáreas ${ }^{8,11,12,14,22,24,25}$, representando essas o principal tipo de cirurgia realizada nos Estados Unidos. Nesse período, ocorreu um incremento de $5,5 \%$ para $22 \%{ }^{25} \mathrm{Com}$ tantas mulheres submetendo-se a cesarianas, a discussão acerca da repetição da tomotocia adquire cada vez maior importância, tendo durante muito tempo se perpetuado o dogma proposto por Craigin ${ }^{5}$ "uma vez cesárea, sempre cesárea"5,8,11,19,20. Entretanto, no início da década de 80, passou a verificar-se um aumento no número de partos vaginais em pacientes cesareadas de aproximadamente $7 \%$ para $18,5 \%^{12,13,17}$. Desde então, numerosos estudos vêm corroborando esse fato, comprovando que o parto vaginal em pacientes com cesárea prévia tem os mesmos resultados perinatais podendo ainda ter melhor prognóstico materno, diminuindo o risco de infecção e aderências ${ }^{1-3,6-16,20,21,23}$.

O fator primordial que leva o tocólogo a uma atitude intervencionista, sem que ocorra uma indicação absoluta de cesariana, seria o risco de rotura uterina ou deiscência da cicatriz uterina. É importante, contudo, mencionar que esses eventos não têm o mesmo significado, pois essa última se manifesta com sangramento transvaginal e sofrimento fetal e pode, em alguns casos, após ter sido consumado o parto, ser resolvida com conduta conservadora, ao passo que a rotura pode ser catastrófica para mãe e feto. No entanto, vários autores relatam altos índices de parto normal em pacientes com cesárea anterior, com baixa incidência dessas complicações ${ }^{1,2,4,6-8,10,11,14,15,22,23,27}$.

Alguns pontos, porém, permanecem controversos, como por exemplo: quantas incisões para cesárea podem ser feitas e se conduzir um parto vaginal com segurança? Após o parto normal deve ser realizada exploração uterina rotineira? Se a mulher foi cesareada por problema recorrente como desproporção céfalo-pélvica, o parto normal deveria ser seguido? Podem ser usadas a analgesia peridural e a ocitocina com segurança nessas pacientes $^{1,7}$ ?

Nesse ponto, convém relembrar os resultados de um estudo multicêntrico de Flamm et al. ${ }^{12}$ no qual foram avaliadas 4.929 pacientes já cesareadas, das quais 1.776 (36\%) submeteramse voluntariamente ao trabalho de parto, utilizando-se ocitocina e a analgesia peridural com as mesmas indicações e contra-indicações das parturientes sem cesárea prévia. Os resultados mostraram boa evolução com parto normal em $74 \%$ dos casos, ressaltando-se que, entre as pacientes com cesárea prévia, 89 já tinham realizado 2 ou 3 histerotomias e que, dessas, $76 \%$ evoluíram com parto vaginal, com indicação em alguns casos do uso de ocitocina, não tendo sido relatado nenhum caso de rotura uterina ${ }^{12}$.

Vários outros autores relatam evolução para parto vaginal em pacientes com cesárea anterior, na maioria dos casos sem ocorrência de finalizações dramáticas, desde que a assistência ao trabalho de parto seja conduzida com monitorização fetal eletrônica e da pressão intra-uterina, com o objetivo de detecção precoce de alguma alteração ${ }^{7,10-13,16,17,22}$. É interessante observar, contudo, que raros trabalhos analisam os fatores prognósticos que estariam envolvidos na predição do parto transpélvico em gestantes cesareadas, uma vez que numerosas variáveis obstétricas podem influenciar 
a via de parto nesse grupo ${ }^{18,21-23,26,27}$. Weinstein et al. ${ }^{26}$ referem que os fatores mais comumente associados com o tipo de parto (transpélvico $\mathrm{x}$ cesariana) são: indicação da cesárea anterior, parto vaginal antes da cesárea anterior, peso fetal no passado e peso atual, escore de Bishop, uso de ocitocina e vários outros ${ }^{26}$. Sua validade, contudo, na predição de um parto vaginal bem sucedido após uma cesariana permanece por ser estabelecida.

Tendo em vista a carência de estudos específicos com essa finalidade, o presente estudo foi desenhado com o objetivo geral de determinar os fatores prognósticos para a ultimação do parto por via transpélvica em gestantes com cesárea anterior admitidas em trabalho de parto no CAM-IMIP.

Foram definidos como objetivos específicos: 1) determinar a odds ratio de um parto transpélvico para os seguintes fatores: idade materna, paridade, parto vaginal anterior, tempo transcorrido entre a cesárea anterior e o parto atual, local de realização e indicação da cesárea anterior e tipo de histerrorafia prévia; 2) determinar a odds ratio ajustada para cada uma dessas variáveis depois de controlado o papel das variáveis potencialmente confundidoras, através de análise de regressão logística múltipla.

\section{Pacientes e Métodos}

Foi realizado um estudo analíticoobservacional, retrospectivo, tipo caso-controle, incluindo todos os partos, tanto transpélvicos quanto cesarianas, ocorridos em mulheres com cesárea anterior assistidas no CAM-IMIP no período de janeiro de 1991 a dezembro de 1994 , desde que aplicados os critérios de inclusão e exclusão.

Durante o período mencionado, foram assistidos no serviço 16.094 partos, sendo o percentual de cesarianas de $27,4 \%(n=4.412)$ e o total de pacientes com cesárea anterior de 2.956. Essas últimas representaram $18,4 \%$ dos partos no período e $29,5 \%$ do total de cesáreas realizadas no IMIP.

Como casos, foram incluídas as mulheres que evoluíram com parto transpélvico e controles aquelas cuja gestação foi ultimada por cesariana. O tamanho final da amostra, após aplicados os critérios de inclusão e exclusão, foi de 494 mulheres já cesareadas, sendo 338 casos de parto transpélvico e 156 controles submetidas a cesariana.

Só foram incluídas no estudo as pacientes que apresentassem idade gestacional maior ou igual a 36 semanas, parto anterior por cesariana há pelo menos um ano, concepto vivo, trabalho de parto espontâneo e apresentação cefálica fletida.

Foram excluídas todas as pacientes que apresentassem gestações de alto-risco, dentre elas, a doença hipertensiva específica da gestação, diabetes, crescimento intra-uterino retardado e outras doenças maternas associadas; foram ainda excluídos todos os casos de sofrimento fetal anteparto (diagnosticado por meio da alteração das provas de vitalidade fetal); parto transpélvico anterior (quando ocorrido depois da realização da cesárea) e por fim todas as pacientes com indicação de imediata resolução do parto por ocasião do internamento como foram os casos de descolamento prematuro de placenta normoinserida (DPPNI), prolapso de cordão e outros.

\section{Processamento e Análise dos Dados}

Os dados foram coletados mediante formulário-padrão pré-codificado, sendo digitados e armazenados no banco de dados DBASE III PLUS. Esses dados foram analisados por meio dos programas de domínio público Epi-Info 6.0 e Epi-Soft, sendo utilizados os seguintes testes estatísticos: $\chi^{2}$ de associação e teste exato de Fisher e teste " $t$ " de Student para diferença entre médias, considerando-se um nível de significância de 5\% . Odds ratio e seu intervalo de confiança foram determinados como estimativa do risco relativo para os diversos fatores prognósticos estudados e, por último, a análise de regressão logística múltipla foi empregada para controle das variáveis confundidoras.

Para construção dos modelos de regressão logística as variáveis foram definidas como dependente (parto transpélvico) e independentes (idade materna menor que 20 ou a partir de 35 anos, parto vaginal anterior, intervalo entre cesárea anterior e parto atual menor que 2 anos, cesárea anterior no IMIP, indicação da cesárea anterior por complicações da gravidez ou por distocias / discinesias e histerorrafia prévia em pontos contínuos), codificadas como $\operatorname{sim}=1 /$ não=0 para entrada no programa estatístico.

\section{Resultados}

A distribuição das pacientes com cesárea anterior quanto à via de parto é exibida na Tabela 1, observando-se que 68,4\% (338 casos) evoluíram para o parto transpélvico e $31,6 \%$ (156) para interrupção por via abdominal.

A Tabela 2, por sua vez, apresenta, comparativamente, as características pertinentes às pacientes que evoluíram para parto transpélvico 
e operação cesariana, não sendo observada diferença estatisticamente significante com relação à paridade materna, idade gestacional no parto e intervalo médio entre a cesárea e o parto atual, quando comparados os dois grupos. Em relação à idade materna, houve diferença significativa $(p=0,006)$, verificando-se que a chance de um parto transpélvico foi maior em pacientes mais jovens (média de 23,2 \pm 2,4 anos), em contraposição às pacientes com idade mais avançada, que ultimaram seu parto com maior freqüência por via abdominal. Apresentaram ainda diferença estatisticamente significativa: a história de parto vaginal anterior à cesárea, observando-se uma freqüência de $31,7 \%$ no grupo que evoluiu para parto vaginal e apenas $22.4 \%$ entre as pacientes que se submeteram à cesariana $(\mathrm{p}=0,035)$; e, ainda, quando a cesárea anterior foi realizada no IMIP, manifestando-se nessa circunstância uma maior tendência para parto por via abdominal, em torno de $30 \%$ versus $23,4 \%(p=0,047)$ quando a mesma foi realizada em outro serviço.

Tabela 1 - Distribuição das pacientes de acordo com a via de parto na gestação atual.

\begin{tabular}{lcc}
\hline VIA DE PARTO & $\mathrm{N}^{\mathrm{o}}$ & $\%$ \\
\hline Transpélvico & 338 & $68,4 \%$ \\
Cesariana & 156 & $31,6 \%$ \\
Total & 494 & 100,0 \\
\hline
\end{tabular}

Tabela 2 - Características maternas de acordo com o tipo de parto: transpélvico $\mathrm{x}$ cesariana.

\begin{tabular}{lccc}
\hline CARACTERÍSTICA & PARTO & CESARIANA & $\mathrm{p}$ \\
& TRANSPÉLVICO & & \\
\hline Idade materna (média) & $23,2 \pm 2,4$ & $28,9 \pm 2,6$ & 0,006 \\
Paridade (média) & $2,8 \pm 0,6$ & $2,4 \pm 0,4$ & $\mathrm{NS}$ \\
Idade gestacional & $38,3 \pm 1,8$ & $38,5 \pm 1,6$ & $\mathrm{NS}$ \\
Parto vaginal anterior $(\%)$ & $31,7 \%$ & $22,4 \%$ & 0,035 \\
Cesárea anterior no IMIP(\%) & $23,4 \%$ & $30,1 \%$ & 0,047 \\
Intervalo médio entre a cesárea & $2,14 \pm 1,01$ & $2,38 \pm 0,96$ & $\mathrm{NS}$ \\
\multicolumn{1}{c}{ e o parto atual (anos) } & & &
\end{tabular}

NS = não significativo

A Tabela 3 resume as características neonatais dos grupos de estudo, casos e controles, sendo avaliados os seguintes parâmetros: peso do recém-nascido ao nascimento, escores de Apgar no primeiro e quinto minuto, incidência de escores de
Apgar abaixo de sete no quinto minuto e mortalidade perinatal, constatando-se que os resultados não apresentaram diferença significativa entre os grupos supracitados. Faz-se mister mencionar, entretanto, que apesar de não haver diferença entre os dois grupos com relação à freqüência de casos com Apgar menor que 7 no quinto minuto, essa foi mais baixa no grupo com parto transpélvico.

As pacientes que anteriormente haviam sido submetidas à operação cesariana em nosso serviço foram avaliadas, relacionando-se a indicação da cesárea prévia e a evolução atual para parto transpélvico ou abdominal (Tabela 4). Não houve diferença significativa das seguintes indicações em ambos os grupos: desproporção céfalo-pélvica, apresentações anômalas, sofrimento fetal, macrossomia, DPPNI, placenta prévia e outras. As indicações por distocia e/ou discinesias e por complicações da gestação foram mais freqüentes nas pacientes com evolução para parto transpélvico, respectivamente $32,9 \%$ e $30,4 \%$ versus $17 \%$ e $10,6 \%$ naquelas que repetiram a cesariana. Dentre as complicações da gestação, a principal foi a Doença Hipertensiva Específica da Gestação (DHEG).

Tabela 3 - Características neonatais nos dois grupos.

\begin{tabular}{lccc}
\hline CARACTERÍSTICA & $\begin{array}{c}\text { PARTO } \\
\text { TRANSPÉLVICO }\end{array}$ & CESARIANA & $\mathrm{p}$ \\
\hline Peso ao nascer (média em gramas) & $3084 \pm 351$ & $3140 \pm 384$ & $\mathrm{NS}$ \\
\% Apgar $<7$ no 5 $5^{\circ}$ minuto & 0,9 & $1,3 \%$ & $\mathrm{NS}$ \\
Mortalidade perinatal & $0,6 \%$ & $0,6 \%$ & $\mathrm{NS}$ \\
\hline
\end{tabular}

NS = não significativo

Tabela 4 - Indicações da cesárea anterior de acordo com o tipo de parto na gestação atual: transpélvico $\mathrm{x}$ cesariana.

\begin{tabular}{llll}
\hline CARACTERÍSTICA & PARTO & CESARIANA & p \\
& TRANSPÉLVICO & & \\
\hline Distocias/ discinesias & $26(32,9 \%)$ & $08(17,0 \%)$ & 0,051 \\
Complicações da gravidez & $24(30,4 \%)$ & $05(10,6 \%)$ & 0,01 \\
Desproporção céfalo-pélvica & $09(11,4 \%)$ & $05(10,6 \%)$ & NS \\
Apresentação anômalas & $10(12,7 \%)$ & $03(6,4 \%)$ & NS \\
Sofrimento fetal & $08(10,1 \%)$ & $04(8,5 \%)$ & NS \\
Macrossomia & $06(7,6 \%)$ & $04(8,5 \%)$ & NS \\
DPPNI - placenta prévia & $05(6,3 \%)$ & $03(6,4 \%)$ & NS \\
Outras & $04(5,15)$ & $02(4,3 \%)$ & NS \\
\hline
\end{tabular}

NS = não significativo 
Na Tabela 5, está apresentada a odds ratio de parto transpelviano de acordo com os fatores maternos pré-existentes. A partir dessa análise foi observado que o risco permaneceu inalterado para os seguintes fatores: intervalo entre a cesárea anterior e o parto atual menor que 2 anos, o procedimento cirúrgico anterior realizado no serviço, indicação desse por distocias ou discinesias e, por último, a realização da histerorrafia prévia com sutura contínua. Entretanto, houve uma diferença significativa para: idade materna menor que vinte anos e cesárea anterior indicada por patologia da gestação, com odds ratio de 2,07 e 3,67, respectivamente, enquanto que, para idade materna maior que 35 anos, verificou-se odds ratio de 0,54.

Tabela 5 - Associação entre parto transpélvico na gestação atual e fatores maternos pré-existentes.

\begin{tabular}{|c|c|c|}
\hline FATORES MATERNOS & Odds ratio & IC a $95 \%$ \\
\hline Idade $<20$ anos & 2,07 & $1,18-3,66^{*}$ \\
\hline Idade $\geq 35$ anos & 0,54 & $0,36-0,82 *$ \\
\hline Parto vaginal anterior (1 ou mais) & 1,6 & $1,01-2,55$ \\
\hline $\begin{array}{l}\text { Intervalo entre a cesárea anterior } \\
\text { e o parto atual }<2 \text { anos }\end{array}$ & 0,81 & $0,54-1,23$ \\
\hline Cesárea anterior no serviço & 0,71 & $0,45-1,11$ \\
\hline $\begin{array}{l}\text { Cesárea anterior indicada por } \\
\text { complicações da gestação }\end{array}$ & 3,67 & $1,119-12,02 *$ \\
\hline $\begin{array}{l}\text { Cesárea anterior indicada } \\
\text { por distocias/ discinesias }\end{array}$ & 2,39 & $0,09-6,46$ \\
\hline Histerorrafia prévia em pontos contínuos & 1,15 & $0,43-3,11$ \\
\hline
\end{tabular}

significativo

Na Tabela 6 são apresentados os resultados da análise de regressão logística múltipla para identificação das variáveis mais fortemente associadas ao parto transpelviano: no modelo 1 foi determinada a odds ratio ajustada incluindo todas as pacientes estudadas, em um total de 494 casos, sendo estatisticamente significantes a idade materna menor que 20 anos (odds ratio de 1,83) e parto vaginal anterior (odds ratio de 1,88 ). Por outro lado, pacientes com idade de 35 anos ou mais tiveram menor freqüência de parto vaginal (odds ratio de 0,45 ). No modelo 2 foram incluídas apenas as pacientes submetidas a cesárea anterior no serviço (em que se dispunha da informação da indicação), totalizando 126 pacientes, observandose diferença estatisticamente significativa para apenas dois fatores estudados, sendo eles: idade materna igual ou superior a 35 anos (odds ratio $=0,6$ ) e cesárea anterior por patologia da gestação (odds ratio $=2,2$ ).

Tabela 6 - Variáveis associadas ao parto transpélvico (análise de regressão logística múltipla).

Modelo 1

Idade materna $<20$ anos

Idade materna $\geq 35$ anos

Parto vaginal anterior

Modelo 2

Idade materna $\geq 35$ anos

Cesárea anterior por patologia

da gestação
Odds ratio ajustada

1,83

0,45

1,88

Odds ratio ajustada

IC a $95 \%$

0,06

$0,51-0,89$

\section{Discussão}

A antiga proposta de repetir-se sempre a interrupção da gestação por via abdominal em pacientes cesareadas vem decrescendo na última década, baseando-se esta prática mais recente em vários estudos que evidenciam que o parto vaginal pode ser conduzido com segurança e com bons resultados tanto maternos como perinatais $^{1,2,4,6-10,12,13,16-19,21,22}$.

Uma vez que mais de um terço de todas as cesarianas ocorrem como resultado direto de uma cesárea prévia ${ }^{20}$, a realização de uma prova de trabalho de parto nas pacientes já cesareadas, acarretando um percentual de parto transpélvico em torno de $50-70 \%{ }^{20}$, determinaria expressiva redução da taxa global de cesáreas. Paul e Miller ${ }^{20}$ afirmam que, tendo a taxa de cesariana sido estabilizada nos últimos anos, iniciando mesmo um modesto declínio nos Estados Unidos , a maior parte desse declínio é resultado do aumento da prática da prova de trabalho de parto, com parto vaginal bem sucedido após uma cesárea anterior.

Em nosso estudo, realizado em um período de quatro anos, pudemos observar resultados semelhantes, uma vez que $68,4 \%$ das pacientes evoluíram para o parto transpélvico. Embora o atual estudo tenha incluído apenas gestantes de baixo risco, sem patologias associadas, a prova de trabalho de parto tem sido realizada rotineiramente no serviço em toda a população de cesareadas, salvo na presença de contra-indicações absolutas. Assim, conforme descrevemos anteriormente, para o total de pacientes com cesárea anterior atendidas no IMIP no período de 1991 - 1994 ( $\mathrm{n}=2.956)$, o percentual de partos normais foi de 55,8\% (1.652 pacientes), mesmo incluindo-se os casos de alto 
risco, apresentações anômalas e patologias associadas. Verifica-se portanto que foram evitadas 1.652 cesáreas desnecessárias que, se tivessem sido realizadas, elevariam o percentual de cesarianas do serviço de $27,4 \%$ para $37,7 \%$.

Por outro lado, os resultados perinatais foram semelhantes nos dois grupos, não existindo diferença significativa em relação aos escores de Apgar e mortalidade perinatal. Em relação ao prognóstico materno, demonstramos, já em outro estudo realizado em nossa instituição com a mesma população ${ }^{3}$, que as complicações maternas mais importantes ocorreram no grupo submetido a cesariana (infecção, hemorragia), sendo a incidência de deiscência semelhante nos dois grupos $^{3}$. Podemos então afirmar que, além do resultado favorável em termos de diminuição da taxa de cesarianas do serviço, a adoção da prova de trabalho de parto, na medida em que permite um expressivo percentual de partos transpélvicos, determina importante redução da morbidade materna relacionada à cesárea, sem efeitos deletérios para o recém-nascido.

Uma vez definida essa importância da prova de trabalho de parto, persiste a discussão que gestantes se beneficiariam com sua realização sistemática. As indicações de cesariana devem indubitavelmente ser ampliadas na presença de situações anômalas e gestações de alto risco. Para as gestantes de baixo risco, seria importante que o obstetra, ao iniciar uma prova de trabalho, estivesse ciente das chances de sua paciente evoluir ou não para um parto transpélvico, baseado não apenas nos dados objetivos do exame obstétrico, mas em dados da história relacionada com a cesárea anterior.

Existem relatos de uma relação significativa entre algumas variáveis obstétricas que atuariam como fatores prognósticos para a via de parto $^{18,21,22,25,26}$, como o estudo de Weinstein et al. ${ }^{26}$, que apresentam uma análise (realizada em um período de dez anos) objetivando analisar os fatores que influenciariam a evolução para um parto transvaginal em cesareadas. Os autores avaliaram as seguintes variáveis: idade materna, escore de Bishop, paridade, indicação da cesárea anterior e história de parto vaginal anterior, encontrando como fatores preditivos para o parto transpélvico um escore de Bishop igual ou maior que 4, história de parto transvaginal prévio e indicações anteriores de interrupção da gestação como apresentações anômalas e DHEG. A idade materna não apresentou associação significativa com a resolução do parto por via baixa, com uma odds ratio de 0,9 $(\mathrm{IC} \text { a } 95 \%=0,5-1,7)^{26}$.

Os escores de Bishop não foram analisados em nosso estudo, uma vez que o objetivo era determinar variáveis maternas outras que pudessem ajudar o obstetra na decisão por uma ou outra via de parto no momento em que a paciente chegasse ao serviço em trabalho de parto, independente das condições cervicais. Encontramos, todavia, importante associação de um parto transpélvico com êxito com os seguintes fatores: idade materna menor que 20 anos, história de parto vaginal anterior e indicação da cesárea prévia por complicações da gestação.

Esses resultados são semelhantes àqueles descritos por Whiteside et al. ${ }^{27}$, Silver e Gibbs ${ }^{23}$ e Rosen e Dickinson ${ }^{22}$, conquanto nenhum deles descreva a idade como um fator preditivo importante para o parto vaginal. É possível que a idade não constitua um fator favorável per se à parturição espontânea, mas poderia estar associada ao maior empenho do obstetra em tentar o parto transpélvico em adolescentes, sendo menos conservador em gestantes com mais de 35 anos. Por outro lado, a presença de parto vaginal anterior à cesariana apresentou uma forte associação com o êxito na ultimação da gestação por via transpélvica: a odds ratio de via baixa para pacientes com parto vaginal anterior foi de 2,1 na metanálise realizada por Rosen e Dickinson (englobando 29 estudos) e de 1,6 em nossa casuística, persistindo sua importância como variável preditiva mesmo após o controle dos fatores de confusão.

Em relação ao valor prognóstico da indicação da cesárea anterior para o resultado do parto, nossos resultados devem ser interpretados com cautela, uma vez que essas indicações foram determinadas apenas para uma fração da amostra (aquela cujo parto anterior foi assistido no serviço), limitando-se a análise a 126 casos para os quais essa informação estava disponível. Assim, encontramos associação significativa da via baixa com a indicação anterior por patologia da gestação, mas não por apresentação anômala. $O$ pequeno tamanho da amostra aumenta a chance de erro tipo II, podendo uma diferença real não ser detectada pelo estudo, talvez vindo a aparecer em uma amostra maior. Vários autores relataram que a indicação de uma cesárea anterior por apresentações anômalas estaria associada com uma alta taxa de sucesso em uma prova de trabalho de parto na gestação subseqüente ${ }^{22,23,25,26}$.

A interpretação desses fatores prognósticos pode ser de grande auxílio para o obstetra quando esse se defronta com uma paciente com cesárea anterior em trabalho de parto, cabendo-lhe optar pela indicação de cesárea ou, ao invés, pela realização da prova de trabalho de parto. Como uma taxa razoável de sucesso pode ser obtida em um grupo selecionado de pacientes de baixo risco (quase $70 \%$ ), a prova de trabalho de parto deveria ser tentada sempre que não existissem contra- 
indicações à sua realização e fortemente recomendada para as pacientes cuja odds ratio de um parto transpélvico é elevada.

Estudos adicionais são necessários no sentido de, aumentando a população estudada, poder incluir outras variáveis na análise e até mesmo de, seguindo a proposta de Troyer e Parisi ${ }^{24}$, construir um sistema de pontuação para ser aplicado às parturientes com cesárea prévia, o que poderia auxiliar na "seleção adequada das pacientes para repetir a via abdominal ou, ao invés, realizar uma bem-sucedida prova de trabalho de parto" (Weinstein et al. ${ }^{26}$ ).

\section{SUMMARY}

Objectives: to determine prognostic factors for vaginal delivery in pregnant women after previous cesarean section admitted to CAM-IMIP in labor.

Patients and Methods: a case-control study was performed, analyzing all deliveries of patients with previous cesarean section admitted to CAM-IMIP between January 1991 and December 1994. Patients who had a cesarean section $(n=156)$ were considered cases while patients with a vaginal birth were the controls $(n=338)$. Inclusion criteria were: gestational age > 36 weeks, previous cesarean section at least 1 year before, alive fetus, spontaneous labor and vertex presentation. Patients with high-risk pregnancies, acute fetal distress and a previous vaginal delivery after cesarean section were excluded. Statistical analysis was performed with in Epi-Info 6.0 and Epi-Soft, using $\chi^{2}$ test, Fisher's exact test and Student's " $t$ " test. Odds ratio and its 95\% confidence interval was calculated and multiple logistic regression analysis was performed for the control of confounding factors.

Results: overall rate of cesarean section was 31.6\%. Maternal factors significantly associated with vaginal delivery were age $<20$ years $(O R=2.07,95 \% C I=1.18-3.66)$ or $\geq 35$ years $(O R=0.54,95 \% C I=0.36-0.82)$, history of vaginal delivery $(O R=1.6,95 \% C I=1.01-2.55)$ and complications of pregnancy as indication for previous cesarean section $(O R=$ 3.67, 95\% CI =1.19-12.02). A significant association with vaginal delivery could not be detected for other variables: interval between previous cesarean section and present delivery, other indications for cesarean section and type of uterine suture. In a multiple logistic regression model the variables that remained associated with vaginal delivery were maternal age and previous vaginal delivery.

Conclusions: maternal age below 20 years, previous cesarean section indicated due to gestational complications and previous vaginal delivery were favorably associated with vaginal delivery in patients with prior cesarean section. Risk of repeated cesarean section is increased in pregnant women aged 35 years or above. These factors should be contemplated when obstetrical evaluation of the delivery route is performed.

KEY WORDS: Cesarean section. Vaginal delivery. Operative delivery. Resolution of pregnancy.

\section{Referências}

1. Adair CD, Sanchez-Ramos L, Gaudier FL, Kaunitz AM, McDyer DC, Briones D. Labor induction in patients with previous cesarean section. Am J Perinatol 1995; 12: 450-4.

2. Alves MF, Cordeiro A, Cardoso MC, Graça LM. Trial of labor after cesarean section: two years experience. Acta Med Port 1993; 6: 573-6.

3. Carvalho MA, Santos LC, Porto AMF, Azevedo EB, Mesquita C. Via de parto na paciente com cesárea anterior: prognóstico materno e perinatal. Jornada Pernambucana de Ginecologia e Obstetrícia; 1996, 30 de maio a 02 de junho.

4. Chi IC, Whatley A, Wilkens L, Potts M. In-hospital maternal mortality risk by cesarean and vaginal deliveries in two less developed countries. A descriptive study. Int $\mathbf{J}$ Gynaecol Obstet 1986; 24:121-31

5. Craigin E. Conservatism in obstetrics. N Y State $\mathbf{J}$ Med 1916; 104:1-3.

6. Cowan RK, Kinch RA, Ellis B, Anderson R. Trial of labor following cesarean delivery. Obstet Gynecol 1994; 83: 933-6.

7. Comerio D, Crescini C, Idi G, Artuso A, Morganti P, Repetti F. Delivery after previous cesarean section. Experience with 173 patients. Minerva Ginecol 1991; 43: 513-8.

8. Cunningham FG, Mac Donald PC, Gant NF, Leveno KJ, Gilstrap III LC, Hankins GDV, et al. Cesarean delivery and cesarean hysterecomy. In: Ainningham FG, Williams J, editors. 20th ed. Norwalk: Appleton \& Lange; 1997. p. 511-3.

9. Egwuatu VE, Ezeh IO. Vaginal delivery in Nigerian women after a previous cesarean section. Int J Gynaecol Obstet 1990; 32:1-6.

10.Farmer RM, Kirschbaum T, Potter D, Strong TH, Medearis AL. Uterine rupture during trial of labor after previous cesarean section. Am J Obstet Ginecol 1991; 165: 996-1001.

11.Faúndes A, Cecatti JG. Via de parto e mortalidade materna. In: Faúndes A, Cecatti JG, editors. Morte Materna: uma tragédia evitável. Campinas: Editora da UNICAMP; 1991.

12.Flamm BL; Lim OW, Jones C, Fallon D, Newman LA, Mantis JK. Vaginal birth after cesarean section: results of a multicenter study. Am J Obstet Gynecol 1988; 158: 1079-84.

13.Flamm BL, Goings JR, Liu Y, Wolde Tsadik G. Elective repeat cesarean delivery versus trial of labor: a prospective multicenter study. Obstet Gynecol 1994; 83: 927-32 .

14.Fianu S. Maternal mortality in Sweden 1955-1974. Acta Obstet Gynecol Scand 1978; 57:129-31. 
15. Gibbs RS. Clinical risk factors for puerperal infection. Obstet Gynecol 1980; 55 (suppl): 178-84.

16.Hansell RS, McMurray KB, Huey GR. Vaginal birth after two or more cesarean section: a five year experience. Birth 1990; 17:146-50.

17.Holland JG, Dupre AR, Blake PG, Martin RW, Martin JN Jr. Trial of labor after cesarean delivery: experience in the non-university level II regional hospital setting. Obstet Gynecol 1992; 79: 936-9.

18.Jarrel MA, Ashmead GG, Mann LI. Vaginal delivery after cesarean section: a five year study. Obstet Gynecol 1985; 65: 628-32.

19.Martin JN Jr, Morrison JC, Wiser WL. Vaginal birth after cesarean section: the demise of no routine repeat abdominal delivery. Obstet Gynecol Clin North Am 1988; 15:719-36.

20.Paul RH, Miller DA. Cesarean birth: how to reduce the rate. Am J Obstet Gynecol 1995; 72:1903-7.

21.Paul RH, Phelon JP, Yeh SY. Trial of labor in patients with a prior cesarean section. Am J Obstet Gynecol 1985; 151: 297-304.
22. Rosen MG, Dickinson JC. Vaginal birth after cesarean: a meta-analysis of a indicators for success. Obstet Gynecol 1990; 76: 865-9.

23.Silver RK, Gibbs RS. Predictor of vaginal delivery in patients with a previous cesarean section who require oxytocin. Am J Obstet Gynecol 1987; 156: 57-60.

24.Troyer LR, Parisi VM. Obstetric parameters affecting success in a trial of labor: designation of a scoring system. Am J Obstet Gynecol 1992; 167: 1099-104.

25.Volochko A. Comitês mortalidade materna nos Estados Unidos e investigações de mortes maternas na Inglaterra e País de Gales. Seminário Nacional Morbi-Mortalidade Materna; 1989, 2527 agosto; Itapecerica da Serra.

26.Weinstein D, Benshushan A, Tanos V, Zilberstein R, Rojansky N. Predictive score for vaginal birth after cesarean section. Am J Obstet Gynecol 1996;174:192-8.

27. Whiteside DC, Mahan CS, Cook JC. Factors associated with successful vaginal delivery after cesarean section. J Reprod Med 1983; 28: 785-8. 